\title{
Estimate Hand-Finger Position With One Magnetometer and Known Relative Orientation
}

\author{
Zhicheng Yang $^{(}$, Shenggang Yan ${ }^{\circledR}$, Bert-Jan F. van Beijnum ${ }^{\circledR}$, Member, IEEE, Bin Li ${ }^{\circledR}$, \\ and Peter H. Veltink $\left.{ }^{(}\right)$, Senior Member, IEEE
}

\begin{abstract}
Inertial-sensor-based hand motion tracking has become a well-accepted method in many clinical applications, including rehabilitation of the upper extremity. However, major drawbacks are that a sensor on each segment and kinematic chain rule is required. Thus, errors accumulate through the kinematic chain rule. Also, the length of each segment needs to be measured for each user. We propose a novel method in which a permanent magnet on the dorsal side of the hand combined with an inertial and magnetic measurement unit (IMMU) on the fingertip is used to estimate the position of the thumb and index fingertip relative to the position of the dorsal side of the hand. The biggest advantages of the proposed method are that no prior information and kinematic chain rule are needed. Besides, the required number of sensors is low. A Levenberg-Marquardt (L-M) approach is presented to estimate the relative positions with the known orientations. The performance is demonstrated in multiple experiments in which various movement tasks were performed. The most complex task in which participants performed reaching and grasping movements based on the action research arm test (ARAT) resulted in a median distance error between thumb and index finger of $9.6 \%$.
\end{abstract}

Index Terms-Known orientation, localization, one magnetometer.

\section{INTRODUCTION}

$\mathbf{H}$ AND-FINGER motion tracking has many useful applications, including augmented reality (AR), virtual reality (VR), and especially physical rehabilitation [1]-[3]. Stroke patients or people who suffer from spinal cord injury need rehabilitation to regain full or partial functional recovery, including hand and finger dexterity [4]. The state of hand recovery can be determined by evaluating the relative motion between hands and fingers, such as the range of motion (ROM) of the hand, speed, and accuracy [5]. Currently, the relative

Manuscript received April 5, 2021; accepted April 29, 2021. Date of publication May 6, 2021; date of current version May 24, 2021. The work of Zhicheng Yang was supported by the China Scholarship Council (CSC). The Associate Editor coordinating the review process was Dr. Subhas Chandra Mukhopadhyay. (Corresponding author: Zhicheng Yang.)

Zhicheng Yang is with the School of Marine Science and Technology, Northwestern Polytechnical University, Xi' an 710072, China, and also with the Department of Biomedical Signals and Systems, University of Twente, 7522 NB Enschede, The Netherlands (e-mail: z.yang-1@utwente.nl).

Shenggang Yan and Bin $\mathrm{Li}$ are with the School of Marine Science and Technology, Northwestern Polytechnical University, Xi'an 710072, China.

Bert-Jan F. van Beijnum and Peter H. Veltink are with the Department of Biomedical Signals and Systems, University of Twente, 7522 NB Enschede, The Netherlands.

Digital Object Identifier 10.1109/TIM.2021.3077984 motion is usually assessed by the doctors and therapists in the hospital with certain standard movements, such as the Fugl-Meyer or Jebsen-Taylor hand function assessment [6], [7], which are inaccurate and subjective. In order to make the evaluation more accurate and objective, optical tracking systems (OTSs) and inertial measurement unit (IMU)-based systems, including 3-D accelerometers and 3-D gyroscopes, are commonly adopted [8]-[10]. When a 3-D magnetometer is combined with an IMU, the unit is also referred to an inertial and magnetic measurement unit (IMMU). OTSs, such as Vicon motion systems, have a high position accuracy and are often used as reference systems in laboratory environments. However, these systems are expensive, require time-consuming placement of markers on subjects, and have "line-of-sight" problems. These systems measure position directly, and orientation is measured indirectly [11], [12]. In contrast, tracking systems based on IMMUs are inexpensive, have no "lineof-sight" problem, and directly measure angular velocity, acceleration, and magnetic field. More importantly, they are more portable and can be applied outside the laboratory, which is promising for daily and long-term monitoring at home [13], [14]. However, it cannot measure positions directly.

The IMMU-based system is often used to estimate the orientation in 3-D space. One of the biggest challenges is to solve the drift issue. The change of orientation over time can be estimated by integrating gyroscope data. Nevertheless, this does not provide an orientation with respect to the global coordinate system and may drift. The drift can be compensated by the local gravity and geomagnetic field measured by the accelerometer and magnetometer [15], [16]. The compensation can be quite effective when the magnetic disturbance and nongravitational acceleration are small compared with the geomagnetic field and gravity. However, relative position estimation based on IMMUs is more difficult than the orientation estimation. Due to inherent integration drift, position estimation based on strap-down integration cannot be performed with adequate accuracy for periods longer than a few seconds. To overcome this issue, biomechanical information and external position information are often applied. From the biomechanical information perspective, Kortier et al. [13] proposed a method to reduce position drifts by exploiting a biomechanical model of the hand. Subsequently, Salchow-Hömmen et al. [17] improved the ability to 


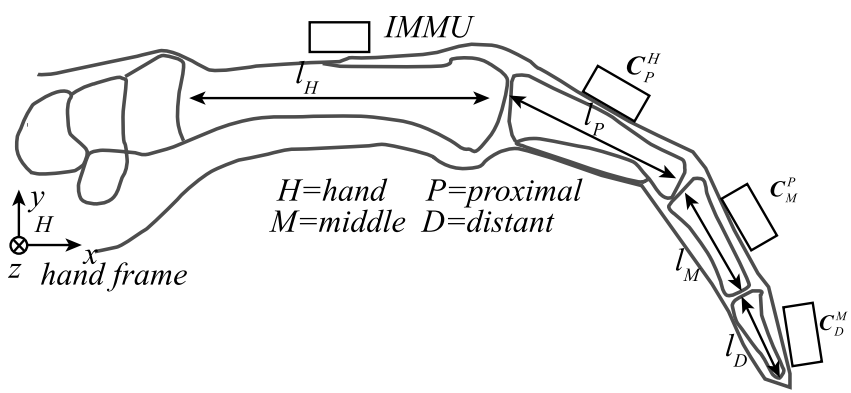

(a)

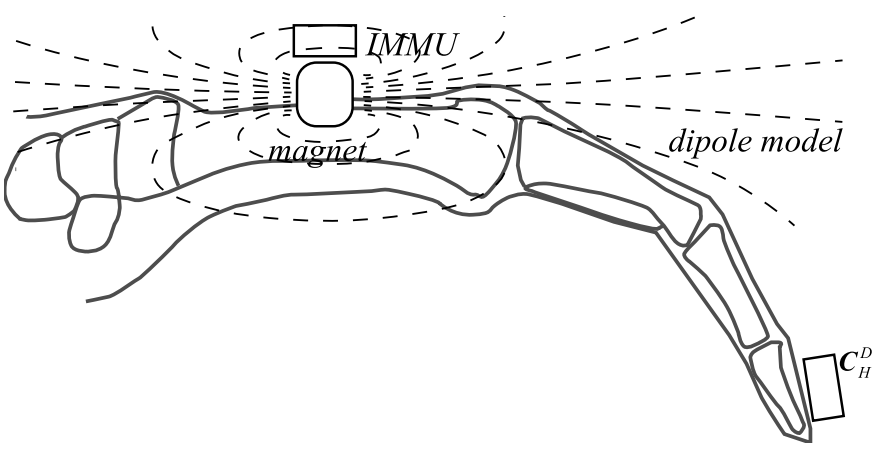

(b)

Fig. 1. Comparison of the configurations between the traditional method and the proposed method. (a) Configuration of IMMUs on the hand with traditional methods. (b) Configuration of IMMUs on the hand with the proposed method.

resist magnetic disturbances by simplifying 3-D rotational movements of segments into 2-D rotational movements.

For the two methods above, the fingertip position was estimated with the biomechanical chain rule, as shown in Fig. 1(a)

$$
\boldsymbol{y}_{\mathrm{tip}, \mathrm{pos}}^{h}=\boldsymbol{l}_{H}^{H}+\boldsymbol{C}_{P}^{H}\left(\boldsymbol{l}_{P}^{P}+\boldsymbol{C}_{M}^{P}\left(\boldsymbol{l}_{M}^{M}+\boldsymbol{C}_{D}^{M} \boldsymbol{l}_{D}^{D}\right)\right)
$$

where $l_{i}^{i}(i=H, P, M, D)$ represents the segment position in its own frame and $C_{i}^{j}(i, j=H, P, M, D)$ represents the relative orientation between segments. The relative orientation and segment lengths are required for using the biomechanical model. There are three major drawbacks. First, model and orientation errors accumulate through the kinematic chain, resulting in position errors. Second, the segment lengths vary among different people and therefore need to be measured for every person before use, which brings error source and is troublesome for users. Third, an IMU is located on each finger segment, which makes the system complex and should preferably be simplified.

In this article, we propose to use a magnet on the hand as a passive magnetic source and one magnetometer on a fingertip of interest as a receiver to estimate the relative position, given known relative orientation between hands and fingers. There are numerous advantages of using such a passive magnetic localization system. First, there is no need to add additional sensors since magnetometers are embedded in available IMMUs and can be used as receivers. Second, it does not consume power. Third, the intermediate IMMUs are neglected, which makes the volume smaller and more convenient for users. The relative orientation can be estimated by only exploiting IMUs, placed on the dorsal side of the

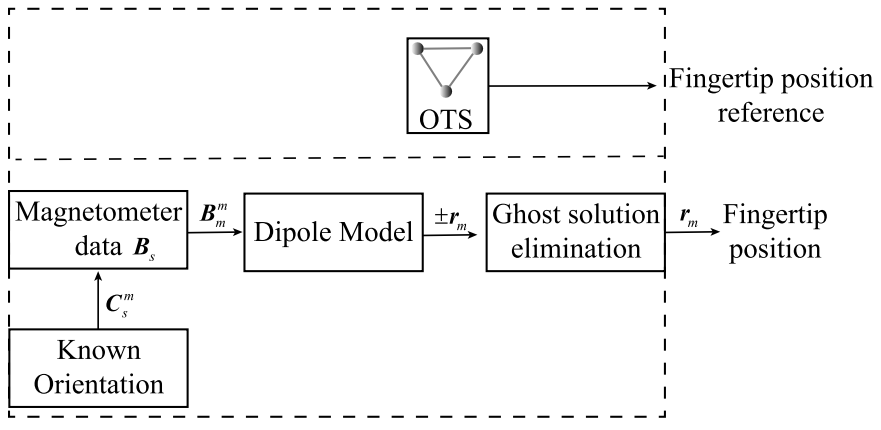

Fig. 2. Flowchart of the proposed method.

hand and the fingertips of interest, which can be found in our previous research [18] or the relative orientation can also be estimated with only IMUs if we assume finger movement as 2DOF [19], [20], such as index finger movements. The aim of this study is to investigate the possibility of estimating relative positions between the hand and several fingertips including thumb and index finger using one magnet on the hand and a single magnetometer on each fingertip of interest, assuming that their relative orientations are known. Thumb and index finger are chosen since they play a more important role compared with rest fingers during daily tasks, such as grasp and pinch [21], [22]. This goal is achieved by analyzing the potentially estimated positions with a dipole model and assuming that the magnetic field generated by the magnet is much stronger than the geomagnetic field.

\section{Methods}

The flowchart of the proposed method is shown in Fig. 2. The magnet on the fingertip is assumed as a magnetic dipole. When the relative orientation between the hand and the fingertip is known, the magnetic field induced by the magnet can be transformed from sensor frame to magnet frame. Subsequently, two possible position estimations are obtained. Through a ghost solution elimination method, the fingertip position relative to the hand is obtained. Finally, the results are compared with the position reference from an OTS.

\section{A. Magnetic Source and Sensor Model}

When a ferromagnetic object is far from the sensor, more than 2.5 times of its length, the object can be approximated as a dipole [23], [24]. As shown in Fig. 3, the magnetic field at $\boldsymbol{r}_{m}$ generated by the magnet (located at the origin) is

$$
\boldsymbol{B}_{m}^{m}=\frac{\mu_{0} M_{m}}{4 \pi r_{m}^{3}}\left[\frac{3}{2} \sin 2 \theta \boldsymbol{e}_{\rho}+\left(3 \cos ^{2} \theta-1\right) \boldsymbol{e}_{z}\right]
$$

where $\mu_{0}$ is the permeability of free space, $M_{m}$ is the magnetic moment, and $\boldsymbol{r}_{m}$ is the position in the magnet frame, which are expressed as follows in a spherical coordinate system:

$r_{m, x}=r_{m} \cos \theta \sin \varphi, r_{m, y}=r_{m} \cos \theta \cos \varphi, \quad r_{m, z}=r_{m} \sin \theta$

where $\left(r_{m}, \theta, \varphi\right)$ gives the radial distance, polar angle, and azimuthal angle and $\boldsymbol{e}_{\rho}$ and $\boldsymbol{e}_{z}$ are projected unit vectors of $\boldsymbol{r}_{m}$ 


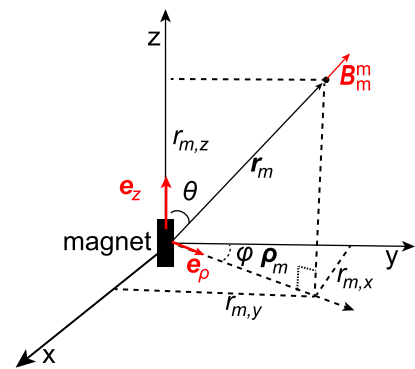

Fig. 3. Dipole model of a magnet. The coordinate system is centered at the center of the magnet, and the $z$-axis is chosen along the magnetic axis. The magnetic field at position $\boldsymbol{r}_{m}$ is $\boldsymbol{B}_{m}$.

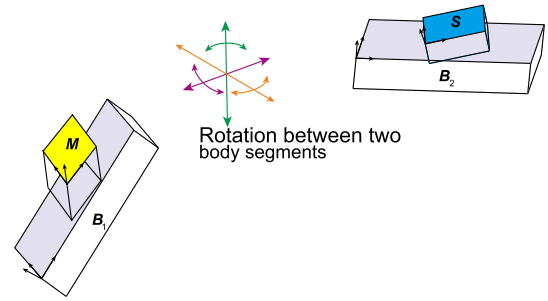

Fig. 4. Relations between two segments. $B_{1}$ and $B_{2}$ represent two body segments. $\boldsymbol{M}$ represents the magnet and $s$ represents the magnetometer.

on the horizontal plane and vertical axis, respectively, as shown in Fig. 3.

When the magnetic field at position $\boldsymbol{r}_{m}$ is measured by a TAM, let it be denoted as $\boldsymbol{y}_{\text {mag }}^{s}$, then this measurement can be modeled as

$$
\boldsymbol{y}_{\text {mag }}^{s}=\boldsymbol{A}\left(\boldsymbol{B}_{m}^{s}+\boldsymbol{B}_{e}^{s}+\boldsymbol{b}\right)+\boldsymbol{n}_{B}
$$

where $\boldsymbol{A}$ is the integrated error parameter including sensitivity errors, nonorthogonality errors, and soft-iron effect errors [25], $\boldsymbol{b}$ is the integrated error including offset errors and hard-iron effect errors, $\boldsymbol{B}_{m}^{s}$ is the magnetic field generated by the magnet, $\boldsymbol{B}_{e}^{s}$ is the disturbing magnetic field including geomagnetic field and surrounding disturbance, and $\boldsymbol{n}_{B}$ is the measurement noise. $\boldsymbol{A}$ and $\boldsymbol{b}$ can be estimated by calibration procedures [26], [27]. When $\left|\boldsymbol{B}_{m}^{s}\right|$ is much stronger than $\left|\boldsymbol{B}_{e}^{s}\right|$, (4) can be approximated as

$$
\boldsymbol{y}_{\mathrm{mag}}^{s} \approx \boldsymbol{A}\left(\boldsymbol{B}_{m}^{s}+\boldsymbol{b}\right)+\boldsymbol{n}_{B} .
$$

\section{B. Relations Between Two Segments}

We place the magnet and magnetometer on two different segments, as shown in Fig. 4. The magnetic field generated by the magnet in the magnet frame can be obtained as

$$
\boldsymbol{B}_{m}^{m} \approx \boldsymbol{C}_{s}^{m}\left(\boldsymbol{A}^{-1} \boldsymbol{y}_{\mathrm{mag}}^{s}-\boldsymbol{b}\right)=\boldsymbol{C}_{B_{1}}^{m} \boldsymbol{C}_{B_{2}}^{B_{1}} \boldsymbol{C}_{s}^{B_{2}}\left(\boldsymbol{A}^{-1} \boldsymbol{y}_{\mathrm{mag}}^{s}-\boldsymbol{b}\right)
$$

where $C_{B_{1}}^{m}$ is the rotation matrix from the segment $B_{1}$ to the magnet, $\boldsymbol{C}_{s}^{B_{2}}$ is the rotation matrix from the magnetometer to the segment $B_{2}, C_{B_{1}}^{m}$ and $C_{s}^{B_{2}}$ can be obtained from sensor to segment calibration, and $C_{B_{2}}^{B_{1}}$ is the rotation matrix between two segments. The position of the magnet in the frame of segment $B_{1}$ is obtained by

$$
\boldsymbol{r}_{B_{1}}=\boldsymbol{C}_{m}^{B 1} \boldsymbol{r}_{m}
$$

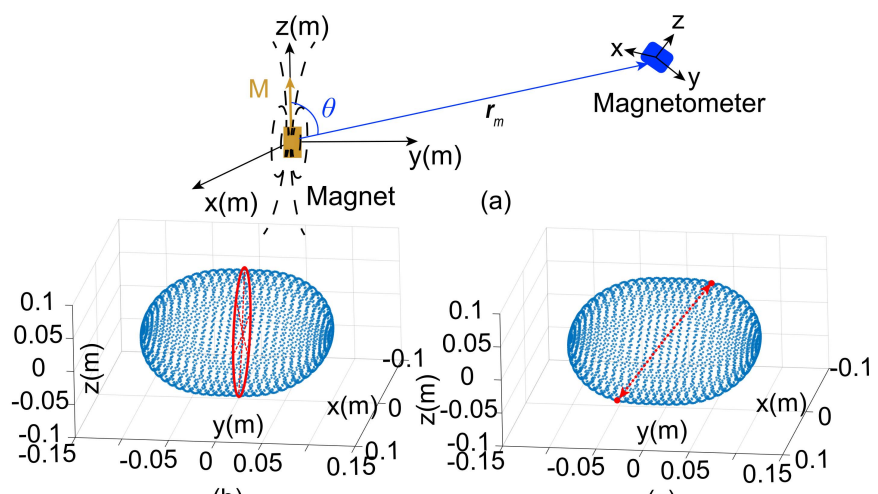

(b)

(c)

Fig. 5. Illustration of the position estimation with one TAM. $\boldsymbol{M}_{m}$ is set as $[0,0,1] A \cdot m^{2}$. (a) Magnet in the center of a coordinate system, with the direction along the $z$-axis. (b) and (c) Possible positions with known orientation $\boldsymbol{C}_{m}^{s}$. For (b), $\boldsymbol{r}_{m} \perp \boldsymbol{M}_{m}$, possible positions are distributed on a circle in 3-D space. For (c), $\boldsymbol{r}_{m}$ is not perpendicular to $\boldsymbol{M}_{m}$, and two possible positions can be estimated as $\pm \boldsymbol{r}_{m}$.

\section{Relative Position Estimation}

1) Existence of Position Estimation: When orientation $\boldsymbol{C}_{m}^{s}$ is known, $\boldsymbol{B}_{m}^{m}$ can be obtained based on (6). Subsequently, $\boldsymbol{r}_{m}$ can be estimated based on $\boldsymbol{B}_{m}^{m}$ and (2). Possible estimations can be categorized into two cases; when $\boldsymbol{M}_{m}$ is perpendicular to $\boldsymbol{r}_{m}$, possible solutions are distributed on a circle in 3-D space, as shown in Fig. 5(b). Otherwise, two possible solutions can be obtained, as shown in Fig. 5(c). The proof can be found in Appendix A.

2) Estimation of $r_{m}$ and $\theta$ : When the orientation $\boldsymbol{C}_{m}^{s}$ is known, the measurement output can be rotated to the magnet frame according to (5). Then, $\boldsymbol{B}_{m}^{m}$ is known based on (5)

$$
\boldsymbol{B}_{m}^{m}=\left(\boldsymbol{C}_{m}^{s}\right)^{-1}\left(\boldsymbol{A}^{-1} \boldsymbol{y}_{\mathrm{mag}}^{s}-\boldsymbol{b}\right) .
$$

Subsequently, the relative position $\boldsymbol{r}_{m}$ with known relative orientation $\boldsymbol{C}_{m}^{s}$ and $\boldsymbol{B}_{m}^{m}$ can be estimated by solving a nonlinear optimization problem based on (2)

$$
\left\{\begin{array}{l}
\left(r_{m}, \theta\right)=\operatorname{argmin}\|\boldsymbol{g}\|_{2}^{2} \\
\boldsymbol{g}=f\left(r_{m}, \theta\right)-\left(B_{m, h}^{m}, B_{m, z}^{m}\right)
\end{array}\right.
$$

where $B_{m, h}^{m}$ and $B_{m, z}^{m}$ arethe horizontal and vertical strength of $\boldsymbol{B}_{m}^{m}$, respectively

$$
B_{m, h}^{m}=\sqrt{\left(B_{m, x}^{m}\right)^{2}+\left(B_{m, y}^{m}\right)^{2}} .
$$

$f\left(r_{m}, \theta\right)$ is obtained based on (2)

$$
f\left(r_{m}, \theta\right)=\frac{\mu_{0} M_{m}}{4 \pi r_{m}^{3}}\left[\frac{3}{2} \sin 2 \theta \boldsymbol{e}_{\rho}+\left(3 \cos ^{2} \theta-1\right) \boldsymbol{e}_{z}\right] .
$$

Then, (9) can be solved by the Levenberg-Marquardt (L-M) method. After initialization for $\left(r_{m}, \theta\right)^{(0)}$, the update can be done using the following equation:

$$
\left(r_{m}, \theta\right)^{(k+1)}=\left(r_{m}, \theta\right)^{(k)}-\left(\lambda \boldsymbol{I}_{2 \times 2}+\boldsymbol{J}_{\boldsymbol{g}}^{T} \boldsymbol{J}_{\boldsymbol{g}}\right)^{-1} \boldsymbol{J}_{\boldsymbol{g}}^{T} \boldsymbol{g}
$$

where $\boldsymbol{I}_{2 \times 2}$ is an unit matrix and $\lambda$ is a damping parameter that would change automatically by the L-M method. When $\lambda$ is small, its performance is close to the Gauss-Newton method. 
Otherwise, its performance is close to the gradient descent method. With the L-M method, there is a greater possibility of convergence when we have a bad initial value. $\boldsymbol{J}_{\boldsymbol{g}}$ is a Jacobian matrix of $g$

$$
\boldsymbol{J}_{\boldsymbol{g}}=\frac{\partial \boldsymbol{f}}{\partial\left(r_{m}, \theta\right)_{m}}=\left[\begin{array}{ll}
\frac{\partial f\left(\left(r_{m}, \theta\right)\right)}{\partial r_{m}} & \frac{\partial f\left(\left(r_{m}, \theta\right)\right)}{\partial \theta}
\end{array}\right]
$$

where

$$
\left\{\begin{array}{l}
\frac{\partial f\left(r_{m}, \theta\right)}{\partial r_{m}}=\frac{-3 \mu_{0} M_{m}}{4 \pi r_{m}^{4}}\left[\frac{3}{2} \sin 2 \theta \boldsymbol{e}_{\rho}+\left(3 \cos ^{2} \theta-1\right) \boldsymbol{e}_{z}\right] \\
\frac{\partial f\left(r_{m}, \theta\right)}{\partial \theta}=\frac{\mu_{0} M_{m}}{4 \pi r_{m}^{3}}\left[3 \cos 2 \theta \boldsymbol{e}_{\rho}-(3 \sin 2 \theta) \boldsymbol{e}_{z}\right] .
\end{array}\right.
$$

3) Estimation of $\varphi$ : In a Cartesian coordinate system, (2) can be rewritten as

$$
\boldsymbol{B}_{m}^{m}=\frac{\mu_{0}}{4 \pi}\left(\frac{3 \boldsymbol{r}_{m}^{T} \boldsymbol{M}_{m} \boldsymbol{r}_{m}}{r_{m}^{5}}-\frac{\boldsymbol{M}_{m}}{r_{m}^{3}}\right)
$$

As shown in Fig. 3, the direction of magnet is along the $z$ axis in magnet frame, $\boldsymbol{M}_{m}=\left[\begin{array}{lll}0 & 0 & M_{m}\end{array}\right]$. Equation (15) can be simplified as

$$
\boldsymbol{B}_{m}^{m}=\frac{\mu_{0} M_{m}}{4 \pi r^{5}}\left[r_{m, x} r_{m, z} \quad r_{m, y} r_{m, z} \quad r_{m, z}^{2}-r_{m}^{2}\right]
$$

Based on (3) and (16), $\varphi$ can be obtained

$$
\tan \varphi=\frac{r_{m, y}}{r_{m, x}}=\frac{B_{m, y}}{B_{m, x}} .
$$

4) Ghost Solution Elimination: From Section II-C3, $\boldsymbol{r}_{m}$ can be estimated either on a circle or two points with additional information $\boldsymbol{C}_{m}^{s} . \boldsymbol{r}_{m}$ lies on a circle only happens when $\theta=\pi / 2$, which very rarely happens and can be prevented by designing the magnet configuration. The goal of this section is to determine solution $\boldsymbol{r}_{m}$ from possible solutions $\pm \boldsymbol{r}_{m}$. For the daily task, positions of the index and thumb fingertips are restricted

$$
\boldsymbol{r}_{f x}>0
$$

where $\boldsymbol{r}_{f x}$ is the position of fingers along the $x$-axis of the hand frame (see Fig. 7 where this direction is defined) and $f$ represents fingers, including the thumb and index finger. Then, $\boldsymbol{r}_{m}$ is uniquely determined. The algorithm proposed and derived above is summarized in Algorithm 1.

\section{Uncertainty of the Estimation}

As shown in (4), the magnetic field generated by the magnet is disturbed by the geomagnetic field, ferromagnetic object, and measurement noise. The sensitivities of estimations to the disturbances vary at different positions, leading to different uncertainties at different positions. Suppose that we have a magnetic disturbance $\boldsymbol{B}_{e}^{m}$ (expressed with $\boldsymbol{e}_{\rho}$ and $\boldsymbol{e}_{z}$ ), and then, an error $\delta \boldsymbol{u}_{m}\left(\delta r_{m}, \delta \theta\right)$ caused by the $\boldsymbol{B}_{e}^{m}$ will be added to the relative position $\boldsymbol{u}_{m}\left(r_{m}, \theta\right)$. Then, we can obtain the following equation based on (11):

$$
\left\{\begin{array}{l}
\boldsymbol{y}_{\mathrm{mag}}^{m} \approx f\left(\boldsymbol{u}_{m}\right) \\
\boldsymbol{y}_{\mathrm{mag}}^{m}+\boldsymbol{B}_{e}^{m} \approx\left(\boldsymbol{u}_{m}+\delta \boldsymbol{u}_{m}\right) .
\end{array}\right.
$$
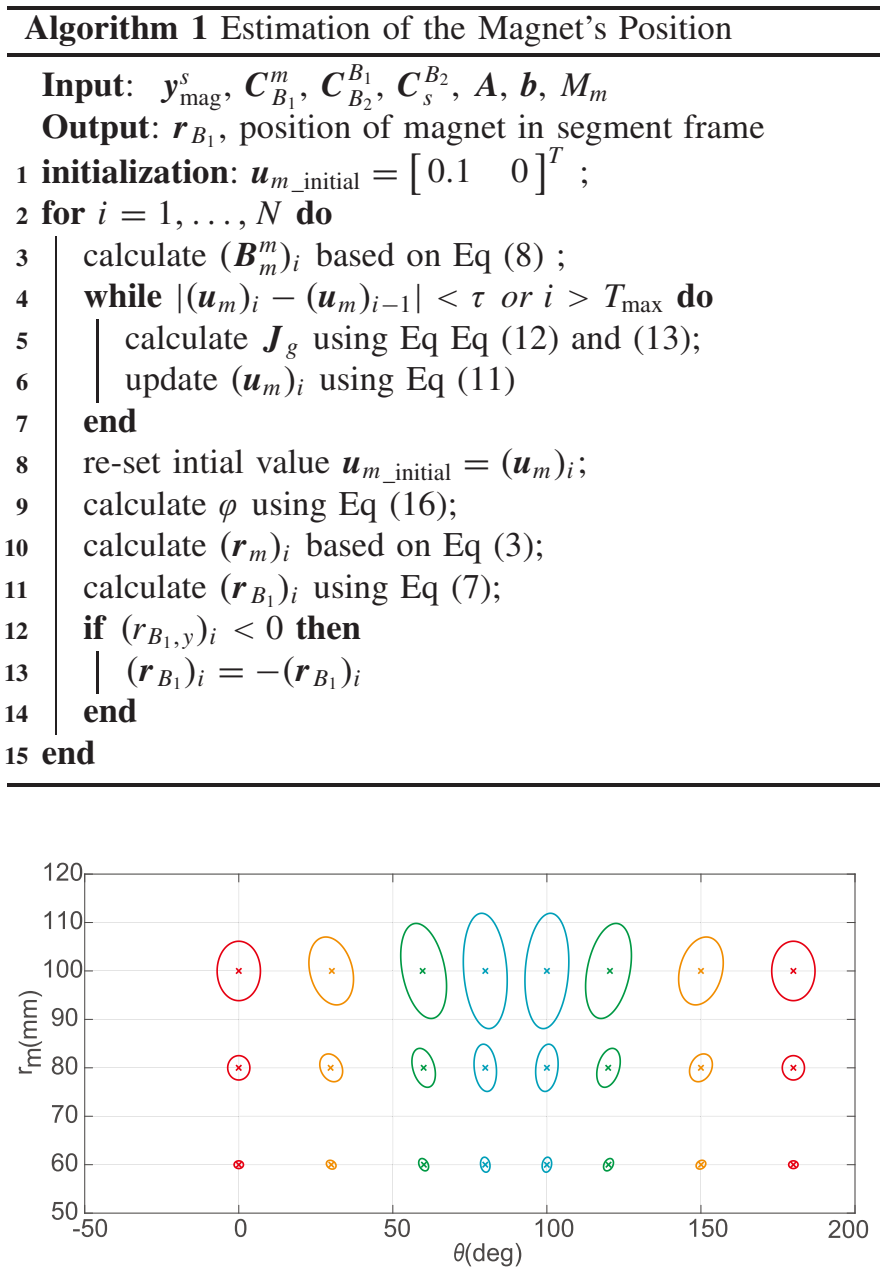

Fig. 6. Simulation of the estimated uncertainty with respect to $r_{m}$ and $\theta$. $\boldsymbol{M}_{m}=\left[\begin{array}{lll}0 & 0 & 1.6\end{array}\right] \mathrm{A} \cdot \mathrm{m}^{2}$, and $r_{m}=60,80$, and $100 \mathrm{~mm} \cdot \theta=$ $0^{\circ}, 30^{\circ}, 60^{\circ}, 80^{\circ}, 100^{\circ}, 120^{\circ}, 150^{\circ}$, and $180^{\circ} . \varphi=0^{\circ}$. The magnetic disturbance is set as a Gaussian distribution with zero mean and 50- $\mu \mathrm{T}$ standard deviation.

Based on (13) and (19), we obtain

$$
\begin{aligned}
\boldsymbol{B}_{e}^{m} & =f\left(\boldsymbol{u}_{m}+\delta \boldsymbol{u}_{m}\right)-f\left(\boldsymbol{u}_{m}\right) \\
& \approx \boldsymbol{J}_{g} \delta \boldsymbol{u}_{m} .
\end{aligned}
$$

The relative position error $\delta \boldsymbol{u}_{m}$ caused by the disturbance $\boldsymbol{B}_{e}^{m}$ can be approximated as

$$
\delta \boldsymbol{u}_{m} \approx \boldsymbol{J}_{g}^{-1} \boldsymbol{B}_{e}^{m} .
$$

According to the error propagation theory, the variance $\boldsymbol{Q}$ of $\delta \boldsymbol{u}_{m}$ can be expressed as

$$
\boldsymbol{Q}=\operatorname{Cov}\left(\delta r_{m}, \delta \theta\right)=\boldsymbol{J}_{g}^{-1} \operatorname{Var}\left(\boldsymbol{B}_{e}^{m}\right)\left(\boldsymbol{J}_{g}^{-1}\right)^{T}
$$

where $\operatorname{Var}\left(\boldsymbol{B}_{e}^{m}\right)$ is the variance of disturbance $\boldsymbol{B}_{e}^{m}$ and $\boldsymbol{Q}$ is a $2 \times 2$ matrix which represents the uncertainty ellipse of the estimation at position $\boldsymbol{u}_{m}$. Fig. 6 shows a simulation result that the uncertainty region caused by a Gaussian disturbance with zero mean and standard deviation $50 \mu \mathrm{T}$. With a certain $\theta$, the uncertainty increases as $r_{m}$ increases. With a fixed distance $r_{m}$, the uncertainty is smaller when $\theta$ approximates $0^{\circ}$ and larger when $\theta$ approximates $90^{\circ}$. 


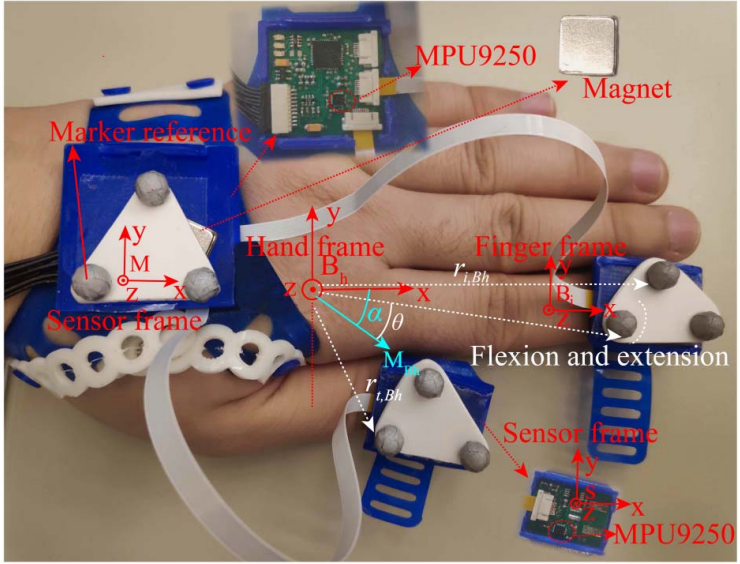

Fig. 7. Experiment setup. Three measurement units were attached to the dorsal side of the hand, index fingertip, and thumb fingertip. A magnet was used as a passive magnetic source, and its size was $11 \mathrm{~mm} \times 11 \mathrm{~mm} \times 11 \mathrm{~mm}$. Three clusters of markers were attached to the surfaces of the measurement setups and the magnet.

\section{EXPERIMENTAL METHODS}

\section{A. Experiment Setups}

The sensor system included three IMMUs ( MPU9250, InvenSense) fixed on the most distal segment of the thumb, index finger, and the dorsal side of the hand. A magnet was also fixed on the dorsal side of the hand, as shown in Fig. 7. The orientation and strength of the magnetic moment needs to be optimized in order to minimize the overall position estimation error. Based on the simulation results, the best option for $\theta$ (angle between $\boldsymbol{r}_{m}$ and $\boldsymbol{M}_{m}$ ) should be between $0^{\circ}$ and $90^{\circ}$ during flexion and extension of the thumb and index finger. As shown in Fig. 7, the angle between $\boldsymbol{M}_{m}$ and $x$-axis is denoted as $\alpha$, which was set as $45^{\circ}$. The strength of the magnet is $3.2 A \cdot m^{2}$. The TAM and accelerometer were sampled synchronously at $100 \mathrm{~Hz}$. All the data were transmitted to a PC through a USB connection. A Vicon system with eight cameras was used and the data were preprocessed with software Vicon Nexus 2.10.2. On each IMMU, a cluster of three markers were placed as shown in Fig. 7, which were used to obtain the relative orientation between the hand and fingers as known orientation $\boldsymbol{C}_{m}^{s}$. The Vicon system also provided the relative position reference for $\boldsymbol{r}_{m}$. The sample frequency of the Vicon system was $100 \mathrm{~Hz}$.

\section{B. Orientation From the OTS}

The known orientation is obtained from clusters of markers. For each cluster, we obtain two vectors $\boldsymbol{r}_{1}, \boldsymbol{r}_{2}$ spanning a configuration of three markers, as shown in Fig. 8. Then, the orientation of the marker frame can be calculated as

$$
\boldsymbol{C}_{m}^{g}=\left[\begin{array}{lll}
\frac{\boldsymbol{n}_{\boldsymbol{r}_{2}} \times \boldsymbol{n}_{\boldsymbol{r}_{3}}}{\left|\boldsymbol{n}_{\boldsymbol{r}_{2}} \times \boldsymbol{n}_{\boldsymbol{r}_{3} \mid}\right|} & \boldsymbol{n}_{\boldsymbol{r}_{2}} & \boldsymbol{n}_{\boldsymbol{r}_{3}}
\end{array}\right]
$$

where $\boldsymbol{n}_{\boldsymbol{r}_{1}}$ and $\boldsymbol{n}_{\boldsymbol{r}_{2}}$ are normalized vectors corresponding to $\boldsymbol{r}_{1}$ and $\boldsymbol{r}_{2}$, respectively. $\boldsymbol{n}_{\boldsymbol{r}_{3}}=\boldsymbol{n}_{\boldsymbol{r}_{1}} \times \boldsymbol{n}_{\boldsymbol{r}_{2}} /\left|\boldsymbol{n}_{\boldsymbol{r}_{1}} \times \boldsymbol{n}_{\boldsymbol{r}_{2}}\right|$. Through the rotation matrix between the OTS coordinate frame and the sensor frame, the orientation assessed by the OTS can be easily transferred to the sensor frame.

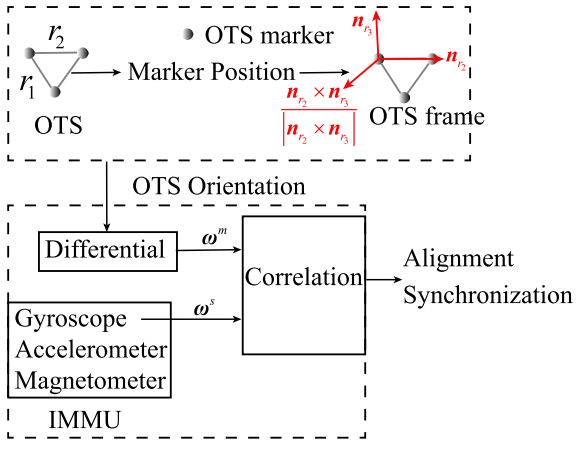

Fig. 8. Alignment and synchronization of the OTS and IMMU system. Angular velocities can be obtained from OTS-based orientation, which can also be measured by the IMMU. The alignment and synchronization were achieved by correlating angular velocities from different frames. The gyroscope was assumed to align with the magnetometer, which can be achieved by calibrations.

\section{Synchronization and Alignment Between the TAM and OTS}

The TAM and OTS were two independent systems, which needed to be synchronized. In addition, the OTS provided known relative orientations for the TAM and therefore needed to be aligned. Here, a gyroscope was used as an intermediate to synchronize and align two systems. The process is shown in Fig. 8.

We obtained angular velocities from the OTS and gyroscope but in different coordinate frames. The synchronization and alignment were achieved by correlating angular velocities in two different coordinate frames. The detailed algorithm can be found in Appendix B.

Since the center of the marker did not coincide with the center of the magnet and TAM, the relative position between the marker and the magnet, as well as the relative position between the marker and TAM, was estimated from the experimental photographs.

\section{Protocols}

The performance of the proposed method was evaluated for different movements (flexion and extension of index finger and thumb, abduction and adduction, and pinch), functional tasks (move an object from position A to positions B, C, and D), and disturbances (geomagnetic disturbance and ferromagnetic object disturbance, see Fig. 9 for the illustration of these experimental cases). Functional tasks are designed based on the action research arm test (ARAT). The experimental protocol consists of a calibration measurement phase followed by five experiments, which are described in the following.

1) Sensor to Segment Calibration: Gyroscopes and accelerometers were used to realize the sensor to segment calibration. The main steps of this procedure are as follows, and more details can be found in Kortier et al.'s work [13].

1) Put the hand flat on the table and static for $10 \mathrm{~s}$, with the back of the hand facing upward, as shown in Fig. 9(a).

2) Put two hands together on the table vertically and hold for $10 \mathrm{~s}$, as shown in Fig. 9(b). 


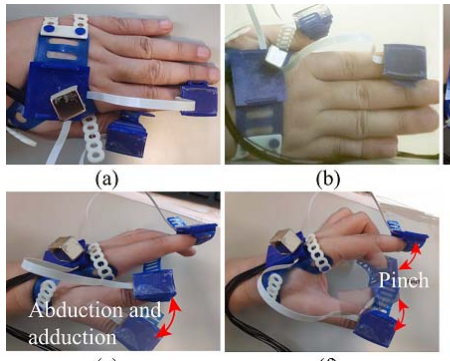

(e)

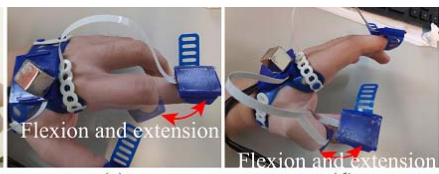

(c)

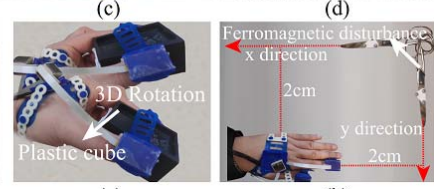

(g)

(h)
Fig. 9. Illustration of the experimental cases. (a) Hand on the table flat. (b) Hand on the table vertically. (c) Index finger flexion and extension. (d) Thumb flexion and extension. (e) Thumb abduction and adduction. (f) Pinch. (g) Hold a plastic cube and rotate in 3-D space, while the relative orientation between the hand and fingers remains unchanged. (h) Move the ferromagnetic object near the setup, while put the hand statically on the surface of the wooden table without any metal bars or screws.

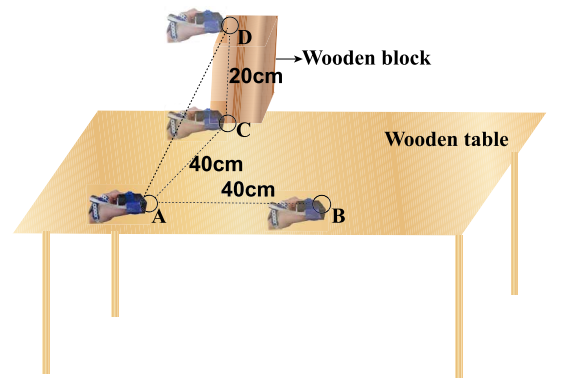

Fig. 10. Illustration of the movements during functional tasks. The participant was asked to grasp the plastic cube from position A to positions B, C, and $\mathrm{D}$ individually. The lengths of $\mathrm{AB}$ and $\mathrm{AC}$ are $40 \mathrm{~cm}$. $\mathrm{AB}$ and $\mathrm{AC}$ are on the surface of the table and they are perpendicular to each other. $C D$ is perpendicular to the surface of the table and $20 \mathrm{~cm}$ long.

3) Flex and extend the index finger repeatedly for $10 \mathrm{~s}$, as shown in Fig. 9(c).

4) Flex and extend the thumb repeatedly for $10 \mathrm{~s}$, as shown in Fig. 9(d).

2) Experiment 1-Joint Movements: The following conditions hold.

1) Stretch the arm with the elbow on the table and then flex and extend the index finger for $20 \mathrm{~s}$, at a rate of three times per second.

2) Repeat (1), with the thumb.

3) Abduct and adduct the thumb at least three times per second for $20 \mathrm{~s}$, as shown in Fig. 9(e).

4) Perform a pinch movement with the thumb and index finger for $20 \mathrm{~s}$, at a rate of three times per second, as shown in Fig. 9(f).

3) Experiment 2-Functional Tasks: The following conditions hold.

1) Put the palm flat on the table near position A for $10 \mathrm{~s}$, as shown in Fig. 10;

2) Grasp the rectangle object with tips of the index finger and thumb from position $\mathrm{A}$ and put it to position $\mathrm{B}$, and then move the hand to the initial position, as shown in Fig. 10;

3) Repeat (1) and (2), but from position A to C.

4) Repeat (1) and (2), but from position A to D.
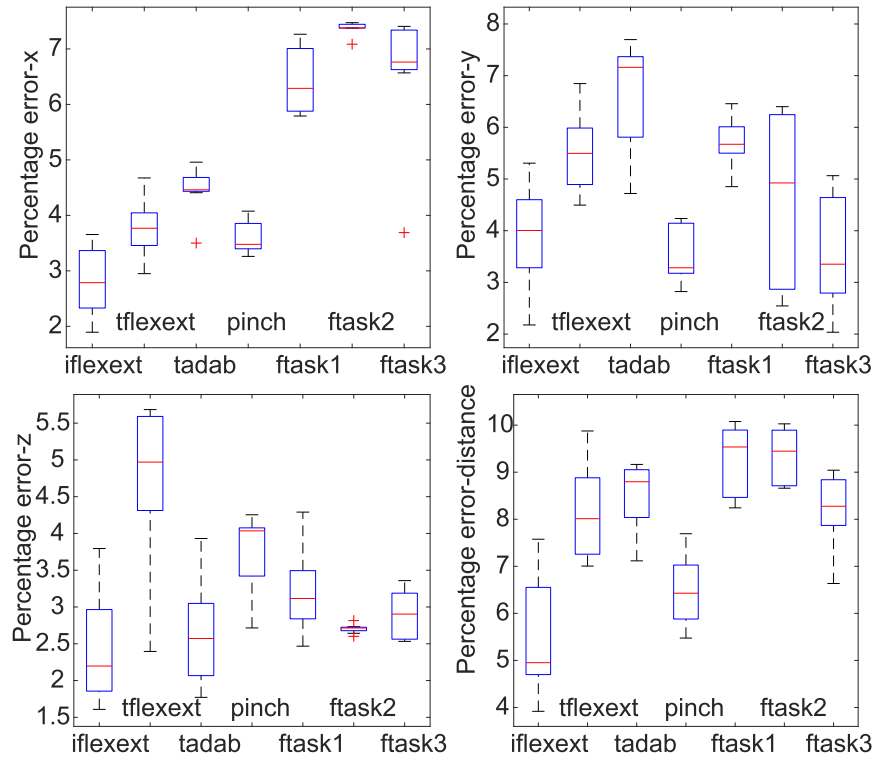

Fig. 11. Relative position errors during different movements evaluated in eight subjects. (a)-(c) Relative position errors along the $x$-, $y$-, and $z$-axis in the hand frame. (d) Relative distance error. iflex, tflexext, and tadab represent index finger flexion and extension, thumb flexion and extension, and thumb abduction and adduction respectively. ftask1, ftask2, and ftask3 represent moving the object from $\mathrm{A}$ to $\mathrm{B}, \mathrm{C}$, and $\mathrm{D}$, respectively, as defined in Experiment 3 (also see Fig. 7).

4) Experiment 3-Influence of Geomagnetic Disturbances: Grasp the rectangle object and rotate the object in 3-D space randomly for $30 \mathrm{~s}$, as shown in Fig. 9(g). During the rotation, do not change the relative orientation and position between the hand and fingers.

5) Experiment 4-Influence of Ferromagnetic Object Disturbances: The following conditions hold.

1) As shown in Fig. 9(h), put the palm flat on the table and keep it static for $10 \mathrm{~s}$.

2) Hold a pair of scissors as a ferromagnetic object and move it along the $x$ - and $y$-axes of the hand frame. The hand frame is shown in Fig. 7, and the trajectories are shown in Fig. 9(h).

Eight participants were involved in Experiments 2 and 3. One participant was involved in Experiments 1, 4, and 5 since the performance of the algorithm was not related to the participants in these experiments. All the experimental data were processed based on Algorithm 1, and the estimated relative position was compared with the Vicon system.

\section{RESULTS}

\section{A. Experiment 1-Joint Movements and Experiment 2-Functional Tasks}

Fig. 11 shows the box plot of median error from eight participants during different movements. Among joint movements, the median distance errors during index finger flexion and extension, thumb flexion and extension, thumb abduction and adduction, and pinch movement are $4.9 \%, 8.0 \%, 8.7 \%$, and $6.4 \%$, respectively. The median distance error during task 1 (move object A to B), task 2 (move object A to C), and task 3 

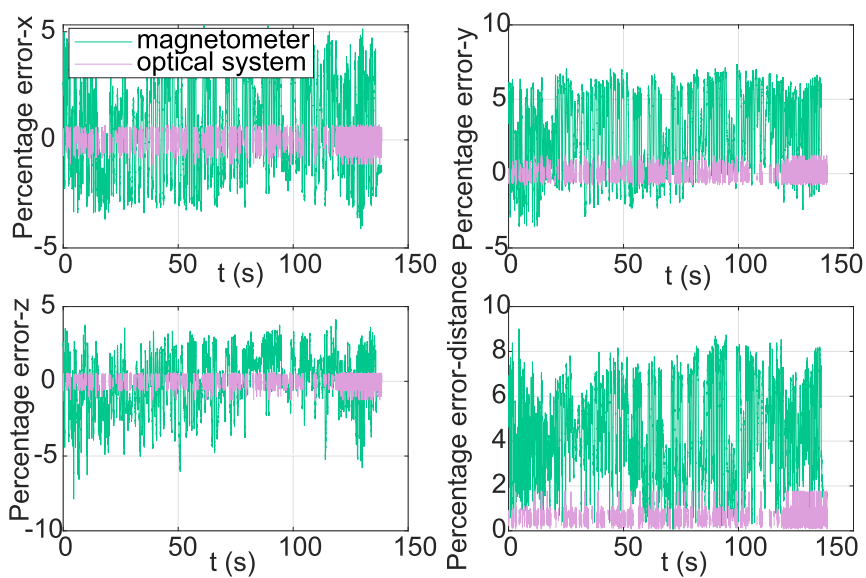

Fig. 12. Relative position errors between the hand and index fingertip during $3-\mathrm{D}$ rotations. The relative orientation between the hand and index finger is constant during rotations.

(move object A to D) are $9.4 \%, 9.3 \%$, and $8.2 \%$, respectively. The discontinuity of the estimation based on OTS is caused by the "line-of-sight" problem.

\section{B. Experiment 3-Influence of Geomagnetic Disturbances}

During the 3-D rotational movements, there are no movements between hand and fingers, and relative position errors caused by the changing geomagnetic field in the sensor frame are shown in Fig. 13. Fingertip position based on the OTS was chosen as a position reference, whose standard deviation was $\left[\begin{array}{llll}0.53 \% & 0.65 \% & 0.53 \%\end{array}\right]$. The standard deviation of fingertip position errors based on magnetometer was $\left[\begin{array}{lll}3.3 \% & 3.9 \% & 2.8 \%\end{array}\right]$ which was caused by the geomagnetic disturbance.

\section{Experiment 4-Influence of Ferromagnetic Object Disturbances}

When the pair of scissors moves approximately along the $x$-axis of the hand frame, the largest estimated distance error was $10.6 \%$, as shown in Fig. 11(a). When the pair of scissors moved approximately along the $y$-axis, the largest distance error is $32.3 \%$, as shown in Fig. 11(b). The distance error is related to the direction of the pair of scissors.

\section{Discussion}

We proposed and evaluated a magnetometer-magnet-based setup for the estimations of interested fingertip position relative to the hand, with the assumption that the orientation of the fingertip relative to the hand is known. The precision of the proposed method is comparable to the precision reported in the literature [13], [17]. To our best knowledge, three studies performed kinematic measurements in human subjects and compared their results with the OTS system. They exploited a similar IMMU-based system with one IMMU attached to each segment of the fingers and a kinematic model of the hand. Compared with Kortier et al.'s and van den Noort et al.'s work [13], [28], Salchow-Hömmen et al. [17] used biomechanical

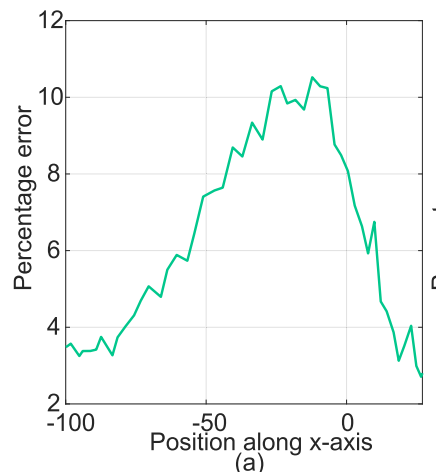

(a)

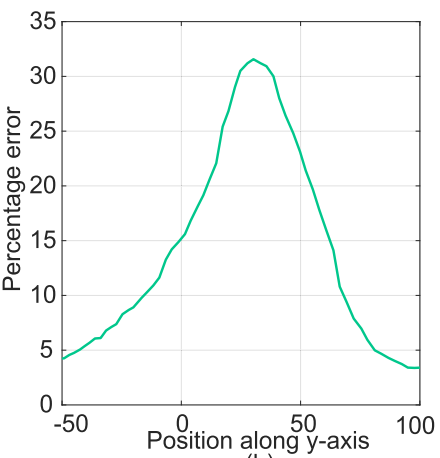

(b)
Fig. 13. Relative position errors between the hand and the index finger in the presence of ferromagnetic disturbances. (a) and (b) Distance error with trajectories of the ferromagnetic object along the $x$-and $y$-axes. The moving direction can be seen in Fig. 9(h).

TABLE I

Distance ERrors of the Estimated Relative Position With the DifFERENT METHODS DURING DiFFERENT MOVEMENTS

\begin{tabular}{cccc}
\hline Items & $\begin{array}{c}\text { Kortier and } \\
\text { Noord et al. }\end{array}$ & $\begin{array}{c}\text { Salchow-Hömmen } \\
\text { et al. }\end{array}$ & $\begin{array}{c}\text { The proposed } \\
\text { method }\end{array}$ \\
\hline iflexext $(\mathrm{cm})$ & 0.5 & 0.5 & 0.6 \\
Pinch $(\mathrm{cm})$ & $1.4 \sim 2.1$ & 1.5 & 0.8 \\
\hline
\end{tabular}

constraints to reduce the influences of magnetic disturbances. The comparison of distance errors during the flexion and extension of the index finger and pinch movement is shown in Table I. Combining our method with a kinematic model could potentially further reduce the relative position estimation error if the model is sufficiently accurate, which is not selfevident. The advantage of not using a kinematic model is that it does not require measurement or estimation of the orientation of the intermediate finger segments between the hand and fingertips.

Compared with the OTS, the estimated position error of fingertip relative to the hand was around $10 \mathrm{~mm}$. It is important to discuss the potential sources of remaining estimation errors. The first source is the geomagnetic field disturbance. Although the geomagnetic field was at least three times smaller than the magnetic field generated by the magnet, it still played an important role in the estimation errors, as shown in Fig. 12. In order to investigate the size of influences, we used the orientation of fingertips from the OTS in its own coordinate frame to obtain the geomagnetic field, then transferred it to the sensor frame, and subtracted it from magnetometer outputs. The median errors reduced from $8.0 \%$ and $4.9 \%$ to $6.2 \%$ and $4.0 \%$ during the flexion and extension of the index finger and thumb. The second error source is the direction of the magnet. The best direction of the magnet that caused the least error for the index finger was $\alpha=0^{\circ}$, while the worst option was $\alpha=90^{\circ}$. $\alpha$ was set as $\alpha=45^{\circ}$ when we considered both the index finger and thumb, which caused unwanted errors for both fingers. Third, we used the positions of markers from the OTS as the relative position reference. However, the centers of the markers did not coincide with the center of the magnet and the center of the magnetometer. 


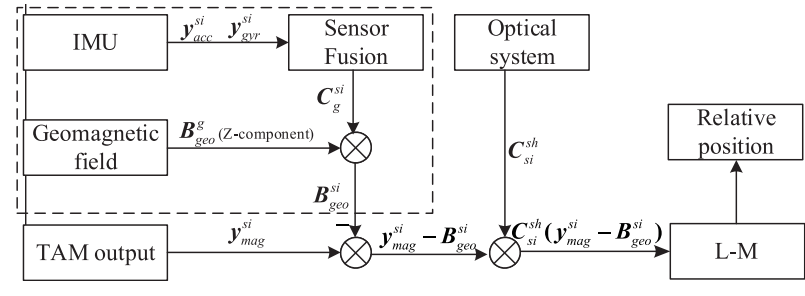

Fig. 14. Illustration of the position improvement by compensating the $z$-axis component of geomagnetic field. $\boldsymbol{B}_{\text {geo }}^{g}$ is the $z$-axis component of geomagnetic field.

Although we roughly estimated the relative position between them, the inaccuracy of this estimation caused errors when the estimation of relative position was compared with the OTS reference. Fourth, the approximation of the magnet as a dipole model caused errors for the relative position estimation. We estimate that the fifth error source is the noise from the magnetometer, which cannot be compensated for by the calibration procedures. The influences of the fourth and the fifth error sources are much smaller compared with previous error sources.

In order to localize a magnetic dipole, at least two TAMs are required without considering the common geomagnetic field, while at least three TAMs are required when including the geomagnetic field. In the latter case, the third TAM is used to measure and subtract the common geomagnetic field [29], [30]. In our research, only one magnetometer is exploited because of the limited space on the fingertip. Therefore, two assumptions are made: 1) the relative orientation is known and 2) the magnetic field induced by the magnet is much stronger than the geomagnetic field. The accuracy of the estimated fingertip positions relative to the hand is closely related to the assumptions. One possible solution to get rid of two assumptions is to add more TAMs. Certain intervals are needed between TAMs in order to measure differential magnetic field with adequate accuracy. If we have two TAMs on the fingertips of interest, the relative orientation is not required. If we have more than three TAMs, neither of the assumptions is required.

When adding new TAMs are not achievable, such as the configuration in our approach, the gyroscope and accelerometer combined with TAM can be used to reduce the impact of the geomagnetic field. As shown in Fig. 14, we obtain the inclination $\boldsymbol{C}_{g}^{\mathrm{si}}$ of the fingertip with the gyroscope and accelerometer, which is compensated by the gravity sensed by the accelerometer and thus does not have drift issues. The fused inclination $\boldsymbol{C}_{g}^{\text {si }}$ can only be used to compensate for the $z$ component of the geomagnetic field in the sensor frame. In this way, the $z$-component of the geomagnetic field disturbance is reduced. The performance of this method depends on the location on earth since the $z$-component of the geomagnetic field varies at different locations.

Compared with Kortier et al.'s and Salchow Hommen et al's work [13], [17], the volume of our setup is larger, especially the components on the fingertips, which happens for two reasons. First, in order to make the system to be easy to wear, we made a 3-D printed coat for the components on fingertips and dorsal side of the hand. However, it made the system larger. Second, our system reserved an interface and related peripheral circuits for the pressure sensor on fingertips. Since we may integrate pressure information of fingertip in the future, which also enlarges the volume of the system. In this work, we were concentrating on the algorithm for the fingertip position estimation relative to the hand and paid less attention to the shape design. In the future, the design of the entire device can be optimized. First, the system can be smaller. Systems in our work and related research [13], [17] exploited the same component MPU9250 (3 mm $\times 3 \mathrm{~mm} \times 1 \mathrm{~mm})$ on the fingertip, which means that the system in this work can be as smaller as others or even smaller after optimization. Second, all the connections inside the system were wired, which were not convenient to users and can be replaced with wireless connections. The sensor data can be transferred by the Bluetooth and so on.

As the strength of the magnet is much stronger than the geomagnetic field, it may cause unwanted forces if it is used near iron objects, such as iron tables or chairs. For a next step, the method to estimate relative positions using a permanent magnet and TAM, assuming known relative orientations, is to be combined with an estimate of relative orientation, as presented in our previous paper [18], in which the relative orientation was estimated based on IMUs only.

\section{CONCLUSION}

A new method was proposed to estimate relative positions between the hand and fingertips of interest with known relative orientations: a magnet on the dorsal side of the hand and an IMMU on the fingertip. The objective of this approach was to avoid using prior information on the length of each segment and reduce the number of IMMUs. The relative position was estimated with the L-M method. During joint movements, the norm of the position error was $4.9 \%, 8.0 \%$, $8.7 \%$, and $6.4 \%$ during index finger flexion, thumb flexion, thumb abduction, and pinch movement respectively. During functional tasks, the distance error was $8.2 \%-9.4 \%$ when the participant grasped a plastic cube from one position to another position. Influences of the geomagnetic disturbance and the ferromagnetic disturbance were investigated. In conclusion, the proposed method is a promising approach for hand motion tracking with comparable performance as previous methods that applied a kinematic model of the fingers and additional IMMU's on intermediate finger segments.

\section{APPENDIX A}

\section{PRoOf of Existence of Position Estimation}

When the orientation $C_{m}^{s}$ is unknown, the strength of the measured magnetic field can be exploited. From (6), we find

$$
\left|\hat{\boldsymbol{y}}_{\text {mag }}^{s}\right|=\sqrt{\left(\boldsymbol{B}_{m}^{m}\right)^{T}\left(\boldsymbol{B}_{m}^{m}\right)}=\frac{\mu_{0} M_{m}}{4 \pi r_{m}^{3}} \sqrt{1+3 \cos ^{2} \theta}
$$

where $\theta$ is the angle between the position $\boldsymbol{r}_{m}$ and the magnetic moment $\boldsymbol{M}_{m} \cdot \hat{\boldsymbol{y}}_{\text {mag }}^{s}$ is

$$
\hat{\boldsymbol{y}}_{\mathrm{mag}}^{s}=\boldsymbol{A}^{-1} \boldsymbol{y}_{\mathrm{mag}}^{s}-\boldsymbol{b} .
$$


There are infinite solutions for position $\boldsymbol{r}_{m}$ with magnetometer output $\hat{\boldsymbol{y}}_{\text {mag }}^{s}$ based on (24), which cannot be uniquely determined. However, the relation between distance $r_{m}$ and the angle $\theta$ is known. Equation (24) represents a surface in 3-D space.

When the orientation $\boldsymbol{C}_{m}^{s}$ is known, the possible solutions can be divided into two cases: whether the magnetic moment $\boldsymbol{M}_{m}$ is perpendicular to the position $\boldsymbol{r}_{m}$.

Case I ( $\boldsymbol{M}_{m}$ Is Perpendicular to $\left.\boldsymbol{r}_{m}\right)$ : In this case, $\theta=\pi / 2$, (2) can be simplified as

$$
\boldsymbol{B}_{m}^{m}=-\frac{\mu_{0}}{4 \pi} \frac{M_{m} \boldsymbol{e}_{z}}{r_{m}^{3}}=-\frac{\mu_{0}}{4 \pi} \frac{\boldsymbol{M}_{m}}{r_{m}^{3}} .
$$

Based on (11), distance $r_{m}$ can be estimated with $\boldsymbol{B}_{m}^{m}$, not the position $\boldsymbol{r}_{m}$. Since positions with the same distance $r_{m}$ share the same magnetic field $\boldsymbol{B}_{m}^{m}$, the possible positions $r_{m}$ degenerate from a surface to a circle in 3-D space with the condition $\boldsymbol{M}_{m} \perp \boldsymbol{r}_{m}$, as shown in Fig. 5(b).

Case II ( $\boldsymbol{M}_{m}$ Is Not Perpendicular to $\left.\boldsymbol{r}_{m}\right)$ : In this case, (2) can be seen as a nonlinear equation set with three equations and three unknown parameters $\boldsymbol{r}_{m}$. Two possible solutions $\pm \boldsymbol{r}_{m}$ can be obtained with $\boldsymbol{B}_{m}^{m}$, as shown in Fig. 3(c). The proof details are as follows.

As the magnetic field measured by the TAM is from the magnet, we know the position of the magnet must exist. The proof equals to: given two magnetic fields $\boldsymbol{B}_{m 1}$ and $\boldsymbol{B}_{m 2}$ that satisfy $\boldsymbol{B}_{m 1}=\boldsymbol{B}_{m 2}$, the possibly corresponding positions $\boldsymbol{r}_{m 1}$ and $\boldsymbol{r}_{m 2}$ have the relation

$$
\boldsymbol{r}_{m 1}= \pm \boldsymbol{r}_{m 2}
$$

Based on (15) and the assumption $\boldsymbol{B}_{m 1}=\boldsymbol{B}_{m 2}$, we obtain

$$
\begin{aligned}
\boldsymbol{B}_{m 2} \times \boldsymbol{r}_{m 2} \cdot \boldsymbol{B}_{m 1} & =-\frac{3\left(\boldsymbol{r}_{m 1}^{T} \boldsymbol{M}_{m}\right) \boldsymbol{M}_{m} \times \boldsymbol{n}_{\boldsymbol{r}_{m 2}} \cdot \boldsymbol{n}_{\boldsymbol{r}_{m 1}}}{r_{m 1}^{4} r_{m 2}^{2}} \\
& =-\frac{3\left(\boldsymbol{r}_{m 1}^{T} \boldsymbol{M}_{m}\right)\left(\boldsymbol{n}_{\boldsymbol{r}_{m 2}} \times \boldsymbol{n}_{\boldsymbol{r}_{m 1}}\right) \cdot \boldsymbol{M}_{m}}{r_{m 1}^{4} r_{m 2}^{2}}=\mathbf{0} .
\end{aligned}
$$

From the assumption, we know $\boldsymbol{r}_{m 1}^{T} \boldsymbol{M}_{m} \neq 0$. Then, we obtain following equation based on (28)

$$
\left(\boldsymbol{n}_{\boldsymbol{r}_{m 2}} \times \boldsymbol{n}_{\boldsymbol{r}_{m 1}}\right) \cdot \boldsymbol{M}_{m}=\mathbf{0} .
$$

Then, $\boldsymbol{M}_{m}$ can be expressed as

$$
\boldsymbol{M}_{m}=k_{1} \boldsymbol{r}_{m 1}+k_{2} \boldsymbol{r}_{m 2}
$$

since $\boldsymbol{M}_{m} \neq \mathbf{0}$ and $k_{1}$ and $k_{2}$ are not zero simultaneously. Combining with (15), we obtain

$$
\begin{aligned}
\boldsymbol{B}_{m 1} & =\frac{\mu_{0}}{4 \pi}\left(\left(\frac{2 k_{1} r_{m 1}^{2}+3 k_{2} \boldsymbol{r}_{m 1}^{T} \boldsymbol{r}_{m 2}}{r_{m 1}^{5}}\right) \boldsymbol{r}_{m 1}-\frac{k_{2}}{r_{m 1}^{3}} \boldsymbol{r}_{m 2}\right) \\
\boldsymbol{B}_{m 2} & =\frac{\mu_{0}}{4 \pi}\left(-\frac{k_{1}}{r_{m 2}^{3}} \boldsymbol{r}_{m 1}+\left(\frac{2 k_{2} r_{m 2}^{2}+3 k_{1} \boldsymbol{r}_{m 2}^{T} \boldsymbol{r}_{m 1}}{r_{m 2}^{5}}\right) \boldsymbol{r}_{m 2}\right)
\end{aligned}
$$

since $\boldsymbol{B}_{m 1}=\boldsymbol{B}_{m 2}$, the following equation is obtained based on (31) and (32):

$$
\left(a_{11} k_{1}+a_{12} k_{2}\right) \boldsymbol{r}_{m 1}-\left(b_{11} k_{1}+b_{12} k_{2}\right) \boldsymbol{r}_{m 2}=0
$$

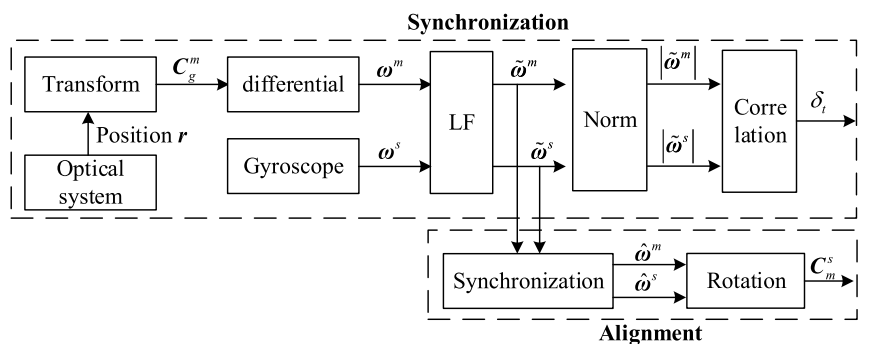

Fig. 15. Illustration of the synchronization and alignment. The angular velocity from the OTS contained high-frequency noise, low-pass filter (LF) was used to reduce the noise. The OTS and gyroscope use the same LF to reduce the time delay of LF. The passband frequency and stopband frequency were set as 8 and $15 \mathrm{~Hz}$, respectively. Alignment was done after the synchronization.

where $a_{11}, a_{12}, b_{11}$, and $b_{12}$ can be expressed as

$$
\begin{cases}a_{11}=\frac{2}{r_{m 1}^{3}}+\frac{1}{r_{m 2}^{3}}, & a_{12}=\frac{3 \boldsymbol{r}_{m 1}^{T} \boldsymbol{r}_{m 2}}{r_{m 2}^{5}} \\ b_{11}=\frac{3 \boldsymbol{r}_{m 1}^{T} \boldsymbol{r}_{m 2}}{r_{m 2}^{5}}, & b_{12}=\frac{2}{r_{m 2}^{3}}+\frac{1}{r_{m 1}^{3}}\end{cases}
$$

if $\boldsymbol{n}_{r 1} \neq \pm \boldsymbol{n}_{r 1}$, we can obtain following equation based on $(33)$ :

$$
\left\{\begin{array}{l}
a_{11} k_{1}+a_{12} k_{2}=0 \\
b_{11} k_{1}+b_{12} k_{2}=0 .
\end{array}\right.
$$

However, (35) only has zero solutions $k_{1}=0$ and $k_{2}=0$ because of following equations:

$$
\begin{aligned}
a_{11} b_{12} & =\frac{5}{r_{m 1}^{3} r_{m 2}^{3}}+\frac{2}{r_{m 1}^{6}}+\frac{2}{r_{m 2}^{6}}>\frac{9}{r_{m 1}^{3} r_{m 2}^{3}} \\
b_{11} a_{12} & =\frac{9 \cos ^{2} \theta}{r_{m 1}^{3} r_{m 2}^{3}}<\frac{9}{r_{m 1}^{3} r_{m 2}^{3}} \\
a_{11} b_{12}-b_{11} a_{12} & >0
\end{aligned}
$$

which is contradict to the assumption that $k_{1}$ and $k_{2}$ are not zero simultaneously. Thus, the assumption $\boldsymbol{n}_{r 1} \neq \pm \boldsymbol{n}_{r 2}$ is not true. On the contrary, we obtain

$$
\boldsymbol{n}_{r 1}= \pm \boldsymbol{n}_{r 2} .
$$

Then, we obtain $\cos ^{2} \theta_{1}=\cos ^{2} \theta_{2}$. Combined with (24), we obtain

$$
r_{m 1}=r_{m 2} \text {. }
$$

Finally, we proof (27) based on (39) and (40).

\section{APPENDIX B \\ SYNCHRONIZATION AND ALIGNMENT}

The synchronization and alignment are done in Fig. 15.

\section{ACKNOWLEDGMENT}

The authors would like to thank the Roessingh Research and Development, Enschede, The Netherlands, for sharing the gait laboratory and the lab manager, Leendert Schaake, for helping with the optical system and the process of data. They would like to thank A. Droog and G.J.W. Wolterink, Biomedical 
Signals and Systems Group, University of Twente, Enschede, The Netherlands, for providing inertial sensor setup and 3-D printed coat for inertial sensors.

\section{REFERENCES}

[1] K. Oka, Y. Sato, and H. Koike, "Real-time fingertip tracking and gesture recognition," IEEE Comput. Graph. Appl., vol. 22, no. 6, pp. 64-71, Nov. 2002.

[2] E. Tunik, S. Saleh, and S. V. Adamovich, "Visuomotor discordance during visually-guided hand movement in virtual reality modulates sensorimotor cortical activity in healthy and hemiparetic subjects,' IEEE Trans. Neural Syst. Rehabil. Eng., vol. 21, no. 2, pp. 198-207, Mar. 2013.

[3] T. Szturm, J. F. Peters, C. Otto, N. Kapadia, and A. Desai, "Taskspecific rehabilitation of finger-hand function using interactive computer gaming," Arch. Phys. Med. Rehabil., vol. 89, no. 11, pp. 2213-2217, Nov. 2008.

[4] O. Lambercy, L. Dovat, R. Gassert, E. Burdet, C. L. Teo, and T. Milner, "A haptic knob for rehabilitation of hand function," IEEE Trans. Neural Syst. Rehabil. Eng., vol. 15, no. 3, pp. 356-366, Sep. 2007.

[5] G. Yavuzer et al., "Mirror therapy improves hand function in subacute stroke: A randomized controlled trial," Arch. Phys. Med. Rehabil., vol. 89, no. 3, pp. 393-398, Mar. 2008.

[6] R. H. Jebsen, N. Taylor, R. B. Trieschmann, M. J. Trotter, and L. A. Howard, "An objective and standardized test of hand function," Arch. Phys. Med. Rehabil., vol. 50, no. 6, pp. 311-319, Jun. 1969.

[7] T. Platz, C. Pinkowski, F. van Wijck, I.-H. Kim, P. di Bella, and G. Johnson, "Reliability and validity of arm function assessment with standardized guidelines for the Fugl-Meyer test, action research Arm test and box and block test: A multicentre study," Clin. Rehabil., vol. 19, no. 4, pp. 404-411, Jun. 2005.

[8] R. Zhu and Z. Zhou, "A real-time articulated human motion tracking using tri-axis inertial/magnetic sensors package," IEEE Trans. Neural Syst. Rehabil. Eng., vol. 12, no. 2, pp. 295-302, Jun. 2004.

[9] G. Ligorio and A. M. Sabatini, "A novel Kalman filter for human motion tracking with an inertial-based dynamic inclinometer," IEEE Trans. Biomed. Eng., vol. 62, no. 8, pp. 2033-2043, Aug. 2015.

[10] J. Schmidt, D. R. Berg, H.-L. Ploeg, and L. Ploeg, "Precision, repeatability and accuracy of optotrak optical motion tracking systems," Int. J. Experim. Comput. Biomech., vol. 1, no. 1, pp. 114-127, 2009.

[11] P. Merriaux, Y. Dupuis, R. Boutteau, P. Vasseur, and X. Savatier, "A study of vicon system positioning performance," Sensors, vol. 17, no. 7, p. 1591, Jul. 2017.

[12] A. Pfister, A. M. West, S. Bronner, and J. A. Noah, "Comparative abilities of microsoft Kinect and vicon 3D motion capture for gait analysis," J. Med. Eng. Technol., vol. 38, no. 5, pp. 274-280, Jul. 2014.

[13] H. G. Kortier, V. I. Sluiter, D. Roetenberg, and P. H. Veltink, "Assessment of hand kinematics using inertial and magnetic sensors," J. NeuroEng. Rehabil., vol. 11, no. 1, p. 70, 2014.

[14] B.-S. Lin, P.-C. Hsiao, S.-Y. Yang, C.-S. Su, and I.-J. Lee, "Data glove system embedded with inertial measurement units for hand function evaluation in stroke patients," IEEE Trans. Neural Syst. Rehabil. Eng., vol. 25, no. 11, pp. 2204-2213, Nov. 2017.

[15] S. O. H. Madgwick, A. J. L. Harrison, and R. Vaidyanathan, "Estimation of IMU and MARG orientation using a gradient descent algorithm," in Proc. IEEE Int. Conf. Rehabil. Robot., Jun. 2011, pp. 1-7.

[16] D. Roetenberg, C. T. M. Baten, and P. H. Veltink, "Estimating body segment orientation by applying inertial and magnetic sensing near ferromagnetic materials," IEEE Trans. Neural Syst. Rehabil. Eng., vol. 15, no. 3, pp. 469-471, Sep. 2007.

[17] C. Salchow-Hömmen, L. Callies, D. Laidig, M. Valtin, T. Schauer, and T. Seel, "A tangible solution for hand motion tracking in clinical applications," Sensors, vol. 19, no. 1, p. 208, Jan. 2019.

[18] Z. Yang, B.-J.-F. van Beijnum, B. Li, S. Yan, and P. H. Veltink, "Estimation of relative hand-finger orientation using a small IMU configuration," Sensors, vol. 20, no. 14, p. 4008, Jul. 2020.

[19] D. Laidig, P. Müller, and T. Seel, "Automatic anatomical calibration for IMU-based elbow angle measurement in disturbed magnetic fields," Current Directions Biomed. Eng., vol. 3, no. 2, pp. 167-170, Sep. 2017.

[20] D. Laidig, D. Lehmann, M.-A. Begin, and T. Seel, "Magnetometer-free realtime inertial motion tracking by exploitation of kinematic constraints in 2-DoF joints," in Proc. 41st Annu. Int. Conf. IEEE Eng. Med. Biol. Soc. (EMBC), Jul. 2019, pp. 1233-1238.
[21] A. Chiri, N. Vitiello, F. Giovacchini, S. Roccella, F. Vecchi, and M. C. Carrozza, "Mechatronic design and characterization of the index finger module of a hand exoskeleton for post-stroke rehabilitation," IEEE/ASME Trans. Mechatronics, vol. 17, no. 5, pp. 884-894, Oct. 2012.

[22] C. E. Lang, S. L. DeJong, and J. A. Beebe, "Recovery of thumb and finger extension and its relation to grasp performance after stroke," J. Neurophysiol., vol. 102, no. 1, pp. 451-459, Jul. 2009.

[23] A. Sheinker, B. Lerner, N. Salomonski, B. Ginzburg, L. Frumkis, and B.-Z. Kaplan, "Localization and magnetic moment estimation of a ferromagnetic target by simulated annealing," Meas. Sci. Technol., vol. 18, p. 3451, Sep. 2007.

[24] C. Wang, X. Zhang, X. Qu, X. Pan, G. Fang, and L. Chen, "A modified magnetic gradient contraction based method for ferromagnetic target localization," Sensors, vol. 16, no. 12, p. 2168 , Dec. 2016.

[25] Z. Yang, S. Yan, and B. Li, "Hybrid calibration method for three-axis gradiometer," IEEE Magn. Lett., vol. 8, pp. 1-5, 2017.

[26] M. Kok, J. D. Hol, T. B. Schön, F. Gustafsson, and H. Luinge, "Calibration of a magnetometer in combination with inertial sensors," in Proc. 15th Int. Conf. Inf. Fusion, Jul. 2012, pp. 787-793.

[27] V. Renaudin, M. H. Afzal, and G. Lachapelle, "Complete triaxis magnetometer calibration in the magnetic domain," J. Sensors, vol. 2010, pp. 1-10, Oct. 2010.

[28] J. C. van den Noort, H. G. Kortier, N. van Beek, D. H. E. J. Veeger, and P. H. Veltink, "Measuring 3D hand and finger kinematics-A comparison between inertial sensing and an opto-electronic marker system," PLoS ONE, vol. 13, no. 2, Feb. 2018, Art. no. e0193329.

[29] H. G. Kortier, J. Antonsson, H. M. Schepers, F. Gustafsson, and P. H. Veltink, "Hand pose estimation by fusion of inertial and magnetic sensing aided by a permanent magnet," IEEE Trans. Neural Syst. Rehabil. Eng., vol. 23, no. 5, pp. 796-806, Sep. 2015.

[30] R. Alimi, N. Geron, E. Weiss, and T. Ram-Cohen, "Ferromagnetic mass localization in check point configuration using a Levenberg Marquardt algorithm," Sensors, vol. 9, no. 11, pp. 8852-8862, Nov. 2009.

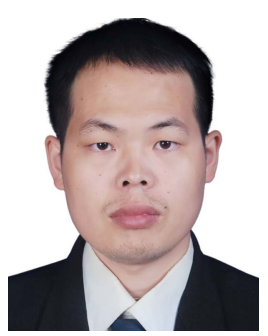

Zhicheng Yang received the B.Sc. degree in automation engineering from Zhengzhou University Zhengzhou, China, in 2013. He is currently pursuing the Ph.D. degree with Northwestern Polytechnical University, Xi'an, China.

In 2018, he joined the University of Twente, Enschede, The Netherlands, as a joint Ph.D. student. His main research interests include motion tracking with inertial sensors, inertial and magnetometer calibration, and localization of ferromagnetic object.

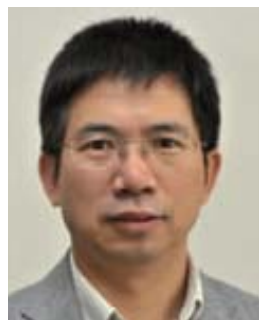

Shenggang Yan received the B.Sc. degree from Central South University, Changsha, China, in 1988 and the M.Sc. and Ph.D. degrees from Northwestern Polytechnical University, Xi'an, China, in 1991 and 2008 , respectively.

$\mathrm{He}$ is currently a Professor with Northwestern Polytechnical University. His main research interests include modern signal processing, high-speed signal processing, and their applications. 


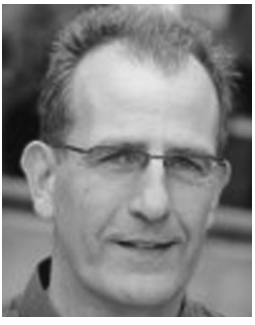

Bert-Jan F. van Beijnum (Member, IEEE) received the M.Sc. and Ph.D. degrees in electrical engineering from the University of Twente, Enschede, The Netherlands.

$\mathrm{He}$ is currently an Associate Professor with the Biomedical Signals and Systems Research Group, University of Twente. His research is embedded in projects of the Centre for Telematics and Information Technology (CTIT) and the Institute of Biomedical Technology and Technical Medicine (MIRA). His research focus is on health systems for chronic diseases and well-being. His research interests include telemedicine, mobile virtual communities for health, smart health systems, smart technologies for decision support, feedback, and coaching.

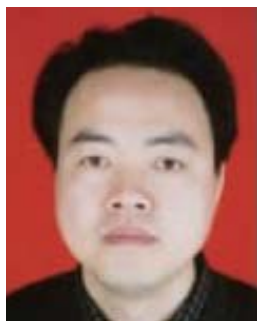

Bin $\mathbf{L i}$ received the B.Sc. and M.Sc. degrees from Northwestern Polytechnical University, Xi'an, China, in 1985 and 1988, respectively.

$\mathrm{He}$ is currently a Professor with Northwestern Polytechnical University. His main research interests include magnetic target detection, sensor technology, and marine electromagnetic field communication.

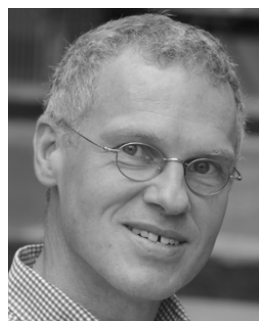

Peter H. Veltink (Senior Member, IEEE) is currently a Professor of technology for the restoration of human function at the University of Twente, Enschede, The Netherlands. He is the (co)author of over 125 peer-reviewed journal articles. He has been the scientific coordinator of three EU research training networks. His research interests include biomechatronics, neural engineering, ambulatory sensing of human movements, artificial human motor control, and neurostimulation.

Prof. Veltink was an Associate Editor of the IEEE TRANSACtions of NeURAL Systems AND ReHAbilitation ENGINEERING until 2014 